\title{
Student difficulties with boundary conditions in the context of electromagnetic waves
}

\author{
Qing X. Ryan* \\ Department of Physics and Astronomy, California State Polytechnic University, \\ Pomona, California 91768, USA \\ Bethany R. Wilcox \\ Department of Physics, University of Colorado, 390 UCB, Boulder, Colorado, 80309, USA \\ Department of Physics, Colorado School of Mines, Golden, Colorado 80401, USA \\ Steven J. Pollock \\ Department of Physics, University of Colorado, 390 UCB, Boulder, Colorado 80309, USA
}

(Received 17 March 2017; revised manuscript received 25 April 2018; published 13 December 2018)

\begin{abstract}
Identifying and understanding student difficulties with physics content in a wide variety of topical areas is an active research area within the physics education research community. Of particular value are investigations of physics topics that appear multiple times in different contexts across the undergraduate physics curriculum. As these common topics reappear, students' difficulties can perpetuate from one context to the next, or new difficulties can emerge as students encounter new physical contexts. One example of such a topic is boundary conditions, which is broadly considered to be an important topic that advanced physics undergraduates are expected to understand and apply in multiple topical areas including classical mechanics, quantum mechanics, and throughout electricity and magnetism. We report findings from an investigation of student difficulties using boundary conditions while focusing on the context of electromagnetic waves. Our data sources include student responses to traditional exam questions, conceptual survey questions, and thinkaloud interviews. The analysis was guided by an analytical framework that characterizes how students activate, construct, execute, and reflect on boundary conditions during physics problem solving. Commonly observed student difficulties include activating boundary conditions in appropriate contexts, constructing a complex expression for the electromagnetic waves, mathematically simplifying and manipulating complex exponentials, and checking if the reflection and transmission coefficients are physical. We also present potential pedagogical implications based on our observations.
\end{abstract}

DOI: 10.1103/PhysRevPhysEducRes.14.020126

\section{INTRODUCTION}

Identifying and understanding student difficulties with physics content in a wide variety of topical areas has been, and continues to be, an active research area within the physics education research (PER) community [1]. Much of this work has focused on students and topics in introductory physics; however, a growing body of research suggests that upper-division students continue to struggle with problem solving even in the advanced physics courses $[2,3]$. Student difficulties in upper-division problem solving stem, in part, from the more complicated math and more

\footnotetext{
*Corresponding author. qxryan@cpp.edu

Published by the American Physical Society under the terms of the Creative Commons Attribution 4.0 International license. Further distribution of this work must maintain attribution to the author(s) and the published article's title, journal citation, and DOI.
}

sophisticated physics characteristic of upper-division content. However, difficulties in the upper division can also stem from the cyclic nature of the physics curriculum, in which some physics topics appear several times in different contexts across the undergraduate curriculum [4]. For these topics, difficulties that are not addressed in early courses can persist, and become worse, when the topic appears again in a more advanced course.

One topic which appears several times throughout the undergraduate curriculum is that of boundary conditions (BCs). In physics, BCs generally refer to the conditions physical quantities must satisfy at the boundary between two regions. These problems are a subset of boundary value problems more generally, which also include problems that require applying initial conditions (i.e., boundary conditions in time) [5]. BCs, and boundary value problems more generally, are ubiquitous in both physics and mathematics. However, BCs are particularly critical in physics because they are often necessary in order to change general and abstract mathematical expressions into physically meaningful 
solutions that have descriptive and predictive power within a particular physical system [5]. BCs are used in a variety of topical areas in physics including classical mechanics, quantum mechanics, and throughout electricity and magnetism. For example, BCs are used when analyzing waves on a finite string in classical mechanics [6]. BCs also appear four separate times in a canonical junior-level electricity and magnetism text (Griffiths Chaps. 3, 6, 7, and 9) [7].

There are relatively few existing research studies investigating student difficulties with BCs. In the context of electrostatics, Wilcox and Pollock [8] looked at student difficulties with separation of variables, where applying BCs is an essential part of the problem solving process. They found that, particularly in the case of separation of variables in spherical coordinates, some students struggled to identify and express the appropriate BCs when they were not explicitly provided in the problem prompt. However, the results from Wilcox and Pollock were limited to students' difficulties with BCs for the scalar potential and did not address students' difficulties with BCs with respect to vector quantities. In the context of quantum mechanics, Singh and Marshman [9] suggested that, while becoming proficient at solving algorithmic problems such as the time-independent Schrödinger equation with complicated BCs, students still struggle to make sense of the material and build a robust knowledge structure. This finding was based on their review of a research study [10] involving analysis of students' sketches of wave functions, as well as explicit questions regarding candidate wave functions in an infinite square well. They documented multiple instances where students failed to examine and apply BCs correctly.

McKagan et al. [11] also touched on BCs in a study of student difficulties with tunneling in quantum mechanics. While applying BCs can be an essential part of solving tunneling problems, their main focus was on student difficulties with the conceptual aspects of the problem, while we focus on the problem-solving process of applying BCs. Zwolak and Manogue [12] also suggested that new difficulties can occur when students transfer certain techniques of solving problems from one context to another. They investigated student reasoning in upper-division electricity and magnetism in the context of the paradigms curriculum at Oregon State University (OSU) [4]. They found that the separation of variables and $\mathrm{BCs}$ procedure did not become a natural problem-solving technique for students when they departed from the quantum context.

In this paper, we investigate student difficulties with applying BCs in the context of electromagnetic (EM) waves. In particular, we focus on BCs as applied to finding relationships between physical quantities for an EM wave incident on a boundary between two linear media. This topic is discussed in Chap. 9 of Griffiths' textbook [7]. Our study contributes to the existing body of knowledge on the use of BCs in this new context-EM waves. The details of BCs investigated in this study are explained in Sec. II.
The findings presented in this paper have some interesting overlap with those of McKagan et al. However, the different physical contexts of these works (i.e., electricity and magnetism vs quantum mechanics) also result in some notable differences in our findings. We will discuss these similarities and differences in more detail in Sec. VII.

The goal of this paper is to investigate common difficulties encountered by upper-division students when utilizing BCs in the context of EM waves (Sec. II). To better organize and structure our investigation of student difficulties, we utilize an existing analytical framework called ACER $[13,14]$ to guide the analysis. The ACER framework is discussed in more detail later in this paper (Secs. III and IV). We then describe the data sources and student population used in this study (Sec. V), and summarize common student difficulties identified with that population (Sec. VI). Finally, we discuss how our results align with related studies of BCs in quantum mechanics (Sec. VII), and end with some implications for teaching, limitations of the study, and suggestions for future work (Sec. VIII).

\section{BOUNDARY CONDITIONS IN THE CONTEXT OF EM WAVES}

In this section, we review the manifestation of $\mathrm{BCs}$ in the context of EM waves, as well as the setup of a typical problem encountered by students in upper-division electricity and magnetism. When an EM wave is incident on a boundary between two linear media, BCs are used to find relationships between physical quantities in the two regions. An example of such a situation is presented in Fig. 1. In this situation, the electric and magnetic fields satisfy boundary conditions derived from Maxwell's equations (see, e.g., Chap. 7 in Griffiths' text [7]):

$$
\begin{aligned}
\vec{E}_{1}^{\|} & =\vec{E}_{2}^{\|}, \\
\epsilon_{1} E_{1}^{\perp}-\epsilon_{2} E_{2}^{\perp} & =\sigma_{f}, \\
B_{1}^{\perp} & =B_{2}^{\perp}, \\
\vec{B}_{1}^{\|} / \mu_{1}-\vec{B}_{2}^{\|} / \mu_{2} & =\vec{K}_{f} \times \hat{n} .
\end{aligned}
$$

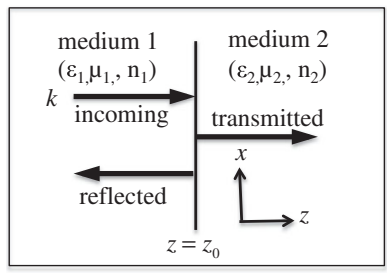

FIG. 1. Setup of a typical BCs problem in the context of EM waves where the boundary is separating medium 1 and 2. $\epsilon_{1}$ and $\epsilon_{2}$ represent the dielectric permittivities of medium 1 and 2 , respectively. $\mu_{1}$ and $\mu_{2}$ represent the permeability constants of medium 1 and 2, respectively. $n_{1}$ and $n_{2}$ represent the indices of refraction of the media. 
Here, I| denotes the parallel component of the field vector, while $\perp$ denotes the perpendicular component of the field vector. Additionally, $\epsilon_{1}$ and $\epsilon_{2}$ represent the dielectric permittivities of medium 1 and 2, respectively; $\mu_{1}$ and $\mu_{2}$ represent the permeability constants of medium 1 and 2 , respectively; $\sigma_{f}$ denotes the free surface charge density at the boundary interface; $\vec{K}_{f}$ denotes the free surface current density at the boundary interface; and $\hat{n}$ is a unit vector perpendicular to the interface (pointing from medium 2 to 1$)$.

In the case of no free charge or current at the boundary, Eqs. (2) and (4) become

$$
\begin{gathered}
\epsilon_{1} E_{1}^{\perp}=\epsilon_{2} E_{2}^{\perp}, \\
\vec{B}_{1}^{\|} / \mu_{1}=\vec{B}_{2}^{\|} / \mu_{2} .
\end{gathered}
$$

Thus, Eqs. (1), (3), (5), and (6) are a complete set of equations when there is no free charge or current at the boundary, which represents the most commonly used situation in the canonical BCs problems that appeared in homework and exam questions investigated by this study.

\section{ANALYTICAL FRAMEWORK}

To guide and structure our analysis of student difficulties with BCs in the context of EM waves, we utilize an analytical framework known as ACER (activation, construction, execution, reflection) [13]. The ACER framework is an analytical tool that was developed to characterize student difficulties with upper-division problem solving by organizing the problem-solving process into four general components: activation of the tools, construction of the model, execution of the mathematics, and reflection on the results. These components appear consistently in expert problem solving $[13,15]$ and are explicitly based on a resources view of the nature of learning $[13,16]$. Since the particulars of using mathematical and physical tools to solve upper-division physics problems are highly context dependent, ACER is designed to be operationalized for specific physics topics. The operationalization of the ACER framework for BCs will be discussed in Sec. IV, and additional details about the ACER framework can be found in Ref. [13].

ACER was originally designed to characterize student difficulties using mathematical tools in physics. The use of $\mathrm{BCs}$, however, would more appropriately be characterized as the application of a physics concept or principle rather than a mathematical tool. Thus, this is a slight extension on the original use of ACER. However, the overarching goal of the ACER framework was to assist in the process of investigating mathematically sophisticated physics problem solving [13]. Thus, since we have found ACER to be a productive analytical tool when thinking about students' difficulties with $\mathrm{BCs}$, and the theoretical grounding of the framework does not preclude the use of a conceptual (rather than mathematical) tool, we have opted to utilize the framework here.

Several other frameworks have been applied to investigations of student difficulties. For example, the framework of epistemic games presented by Tuminaro [17] was developed to analyze students' problem-solving behaviors in terms of locally coherent goal-oriented activities. These games both guide and limit what knowledge students think is appropriate to apply at a given time. Identifying these games provides a way of parsing students' tacit expectations about their approach to solve physics problems [18]. This framework was developed for introductory students and relies on students' explicit discussions about the details of their work. Another framework developed by Bing to analyze upper-level students' uses of mathematics was epistemic framing [19] which refers to students' unconscious answers to the question: "What kind of activity is this?" We can identify students' framings of an activity by examining the justifications and proof students offer to support their mathematical claims [19]. Earlier research on investigating students' understanding includes diSessa's work on epistemology in physics [20,21], Sherin's work on symbolic forms $[22,23]$, and Collins and Fergnson's work on epistemic forms and epistemic games [24].

One significant difference between these theoretical frameworks and ACER is that these frameworks usually require detailed analysis of students' in-the-moment reasonings through video or audio data, while ACER can be used to analyze written solutions - the primary data source for the current work. Connections between these, and other theoretical frameworks and ACER are discussed elsewhere [25]. For the purpose of this study, we are interested in identifying the points in students' work where key difficulties appear. ACER provides a mechanism to identify these points using students' written solutions. The framework also informed the development of interview protocols that targeted more aspects of the problem-solving process and probed deeper into students' reasonings. We developed interview questions to study these common difficulties in more depth (see Sec. V).

\section{ACER OPERATIONALIZATION}

We operationalized ACER for the use of BCs in solving EM wave problems (Fig. 1). The operationalization process involves a content expert working through these problems while carefully documenting their steps and mapping these steps onto the general components of the framework. The resulting outline is discussed with other content experts and refined until consensus is reached that the key elements of the problem have been accounted for. Further refinements are made (if necessary) to accommodate unanticipated aspects of student problem solving when applying this coding scheme to students' work. A skeletal summary of the operationalized framework is listed below. A detailed 
description of the operationalization process is discussed elsewhere [13].

Activation of the tool involves identifying $\mathrm{BCs}$ as a relevant physical tool. Activation can be strongly influenced by the nature of the prompt for a given question. Based on the nature of the prompt, evidence of activation of BCs would be as follows:

A1: Direct cue: use of BCs when explicitly asked to but without being provided with the equations. Note: a question that not only gives students an explicit prompt but also lists all the equations of BCs [Eqs. (1), (3), (5), and (6)] would be considered as having bypassed activation.

A2: Use of BCs when presented a physical situation involving two media and asked for the relationship between physical quantities across the boundary.

Construction of the model involves mapping boundary conditions onto the specific physical situation or system. We operationalized construction into three elements. The numbering of these elements is only for labeling purposes and does not necessarily indicate the order of the problemsolving process.

C1: Write mathematical expressions of the complex fields for the incoming, reflected, and transmitted waves (hereafter denoted by the subscripts: $I, R$, and $T$ ).

$\mathrm{C} 2$ : Select the appropriate BCs to be used with the corresponding components of the fields, which involves knowing the geometry of the situation (e.g., which component of the fields is the parallel or perpendicular component).

C3: Set up equations at the boundary which includes superposing the fields of the incoming and reflected waves, and applying the equations only at the boundary.

Execution of the mathematics involves performing mathematical steps to reduce the result to a form that can be interpreted.

E1: Further simplify the expression after setting it up at the boundary, including regrouping or canceling exponential terms. (Note that the manipulation of complex exponentials here could be its own full investigation. However, identifying student difficulties with complex exponentials is not the focus of this study and, therefore, we did not suboperationalize the ACER framework for the use of complex exponentials by itself.)

E2: Calculate the reflection and transmission coefficient using the amplitudes of the electric field.

Reflection on the results involves reflecting on, and evaluating, the mathematical results to gain physical insight and ensure consistency - a practice common among content experts. There are two common ways to reflect on BCs problems:

R1: Check the units of the relevant quantities.

R2: Check limiting behaviors. (e.g., the reflection coefficient $R$ and transmission coefficient $T$ must satisfy these conditions: $0 \leq R \leq 1,0 \leq T \leq 1, R+T=1$ ).

\section{DATA SOURCES}

Data for this study were collected from the junior-level electrodynamics course at the University of Colorado Boulder (CU). The electrodynamics course at CU (electricity and magnetism II: Griffiths [7] Chaps. 7-12) is the second semester of the electricity and magnetism sequence. The student population is composed of physics, astrophysics, and engineering physics majors with a typical class size of 30-60 students. At CU, electricity and magnetism II is often taught with varying degrees of active engagement through the use of research-based teaching practices [26], such as peer instruction [27] and in-class tutorials [26].

There are three major data sources for this study. First, students' solutions to traditional midterm exams (four semesters) from CU $(N=218)$. Second, student responses to one open-ended question (Fig. 2) on the Colorado UppeR-division ElectrodyNamics Test (CURrENT: a conceptual assessment given to many students in electrodynamics) $[28,29]$ ( $N=500$ from 9 institutions). The third data source was two sets of think-aloud interviews $(N=7)$ with CU students. In the rest of this section, we discuss each of these data sources in detail, noting which elements of the operationalized ACER framework are targeted by each source. Table I summarizes this discussion and outlines each individual ACER element and its corresponding data sources.

Five sets of exam data from four different semesters were collected for this study. We outline the number of solutions from each exam set in Table II. Two instructors taught the class over four semesters. One was a PER faculty member who is also one of the authors of this paper (S. J.P) (exam sets: ES3\&4), the other was a physicist who used some of the materials developed by the PER group such as clicker questions, in-class tutorials, and homework or exam problems (exam sets: ES1, 2\&5). The exam questions analyzed below all posed a situation involving electromagnetic

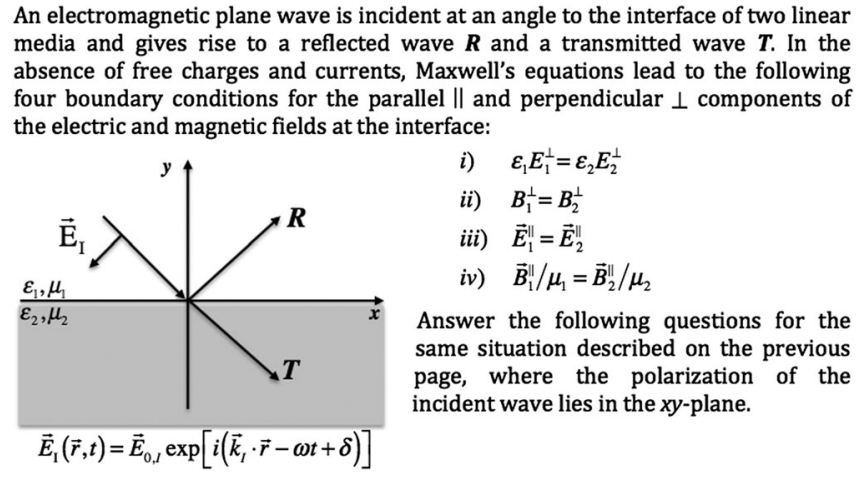

FIG. 2. Question on the CURrENT: Which of these four equations (i-iv) refers to the $y$ component of the electric field at the interface? If this is true for more than one of the equations, then say so. Briefly explain your answer. Note that in the assessment, bold letters $R$ and $T$ were used in the graph to represent the reflected and transmitted wave, instead of $\vec{E}_{R}$ and $\vec{E}_{T}$. 
TABLE I. Corresponding data source and number of solutions for each ACER element (ES refers to "exam set," IS refers to "Interview set").

\begin{tabular}{|c|c|c|c|c|c|c|}
\hline ACER category & ACER subelements & Question details & & $\begin{array}{l}\text { Exam questions } \\
\qquad(N=218)\end{array}$ & $\begin{array}{l}\text { Diagnostic test } \\
(\text { CURrENT) } \\
(N=500)\end{array}$ & $\begin{array}{l}\text { Interview } \\
\text { sets } \\
(N=7)\end{array}$ \\
\hline Activation & $\begin{array}{l}\text { A1: Direct cue-without equations } \\
\text { A2: Boundary presented }\end{array}$ & & & 60 (ES5) & & 3 (IS1) \\
\hline \multirow[t]{2}{*}{ Construction } & C1: Complex expression & $\begin{array}{l}\text { Direction of } \vec{k}, \vec{E}, \vec{B} \\
\vec{k} \operatorname{dot} \vec{r}\end{array}$ & Normal & $\begin{array}{c}108(\mathrm{ES} 3 \& 5) \\
128(\mathrm{ES} 1-4)\end{array}$ & & \\
\hline & $\begin{array}{l}\text { C2: Select appropriate equations } \\
\text { C3: Set up at the boundary }\end{array}$ & $\begin{array}{l}\text { Normal } \\
\text { Oblique }\end{array}$ & Oblique & $\begin{array}{c}36(\mathrm{ES} 1) \\
128(\mathrm{ES} 1-4) \\
36(\mathrm{ES} 1) \\
128(\mathrm{ES} 1-4)\end{array}$ & 500 & 3 (IS1) \\
\hline Execution & $\begin{array}{l}\text { E1: Simplify at the boundary } \\
\text { E2: Mathematical calculation }\end{array}$ & & & $\begin{array}{l}92(\mathrm{ES} 2 \& 3) \\
60(\mathrm{ES} 5)\end{array}$ & & 4 (IS2) \\
\hline Reflection & $\begin{array}{l}\text { R1: Check units } \\
\text { R2: Check limiting behaviors }\end{array}$ & & & $30(\mathrm{ES} 4)$ & & $\begin{array}{l}4 \text { (IS2) } \\
4 \text { (IS2) }\end{array}$ \\
\hline
\end{tabular}

waves at the interface of two media and required using BCs. An example of such a situation is presented in Fig. 1. Exam questions included both normal and oblique incidence situations. For example, one question in exam set 1 (ES1) asks: "Apply the boundary conditions for the electric field given above to write down a mathematical expression that relates the amplitudes of the electric fields of the incident, reflected and transmitted waves." Exams were analyzed by coding common student ideas and/or difficulties that appeared for each element of the operationalized framework. Since different questions targeted different ACER components (i.e., some questions only targeted activation, some questions only targeted construction), not all aspects of the ACER framework were completely captured by exam questions (Table I). To address all aspects of the ACER framework, and to incorporate multiple data sources to further gain insights into students' reasonings, we relied further on data from CURrENT and interviews.

The CURrENT [28] is a research-based assessment that was developed to assess students' conceptual understanding of electrodynamics. This assessment can be accessed at

TABLE II. Exam sets and number of solutions from four different semesters.

\begin{tabular}{lccc}
\hline \hline $\begin{array}{l}\text { Exam } \\
\text { sets (ES) }\end{array}$ & $\begin{array}{c}\text { Semesters } \\
(\mathrm{S})\end{array}$ & $\begin{array}{c}N \\
\text { (solutions) }\end{array}$ & \multicolumn{1}{c}{ Type of question } \\
\hline ES1 & $\mathrm{S} 1$ & $N=36$ & $\begin{array}{c}\text { Both normal \& oblique incidence } \\
\text { (BCs for } \vec{E})\end{array}$ \\
ES2 & S1 & $N=44$ & $\begin{array}{c}\text { Normal incidence (BCs for } \vec{B}) \\
\text { ES3 }\end{array}$ \\
ES4 & S2 & $N=48$ Normal incidence (BCs for $\vec{E}$ ) \\
ES5 & S4 & $N=30 \begin{array}{r}\text { Reflection \& Transmission } \\
\text { coefficient } \\
\text { Normal incidence ( } \omega \text { across the } \\
\text { boundary, and transmission } \\
\text { coefficient) }\end{array}$ \\
\hline \hline
\end{tabular}

the website in Ref. [30]. Validity and reliability studies for this assessment have been conducted [29]. One of the questions on the CURrENT (Fig. 2) asks which of the four given equations refers to the $y$ component of the electric field at the interface and also asks students to provide reasoning. The total number of free-response solutions for this data set is 500 with $38 \%$ from CU students $(N=191)$, $49 \%$ are from 3 other Ph.D. granting institutions $(N=242)$, and $13 \%$ are from 5 other bachelor's degree granting institutions $(N=67)$.

Think-aloud interviews were conducted in two sets, one performed about six months after the other. Interview set 1 included two questions: one targeting ACER element A1 (direct cue-without equations), and one targeting element $\mathrm{C} 2$ (oblique incidence). Interview set 2 included two questions targeting element E2 (execution of mathematics), element R1 (check units), and element R2 (limiting behavior). Interviewees were paid volunteers who completed electricity and magnetism II one or two semesters earlier and responded to an email request for research participants. All interviewees completed the course during one of the semesters for which we have exam data. Final course grades for interview participants were mostly A's and B's (3 A's, 1 A-, 2 B's, and 1 C-).

The purpose of these think-aloud interviews was, in part, to target certain aspects of the ACER framework not accessed by the written exams. For example, interview sets $1 \& 2$ (IS1 \& IS2) were designed to target both elements A1 (direct cue) and R1 (check units) of the ACER framework (Table I). Another purpose of the interviews was to more deeply probe some difficulties identified in the exam solutions. The written solutions provide information on the prevalence and frequency of student difficulties but offer less insight into the thinking and reasoning mechanism behind the students' responses. For example, we observed from written solutions that students have difficulties within 
the execution component of the ACER framework; however, we often cannot determine if those mistakes were caused by not knowing complex exponential math or by simple algebraic errors. Therefore, interview set 2 (IS2) was designed to probe this question.

At the beginning of the interview, the interviewer explained to the students what to expect: "This is a think-aloud interview where you verbalize your thinking while working through the question. I will try not to interrupt and let you do most of the talking. I know thinking-aloud probably does not come naturally to many people, so if you forget to talk, I might remind you to tell me what you are thinking. Or I might ask clarifying questions. Don't be alarmed if I ask you a question, it does not mean you did something wrong, it is simply because I am interested in your reasoning." When the interviewer interacted with the students with questions, the dialog followed a Piaget-style [31] clinical interview method. The interview questions will be discussed in greater detail later in the paper.

\section{RESULTS}

Using the operationalized ACER framework, we identified common student difficulties with BCs in the context of EM waves. To organize the presentation of these difficulties, we group them according to the ACER components. Since different questions or data sources targeted different parts of the ACER framework (Table I), different numbers of solutions will be reported throughout the results section. For exam data, we are reporting the number of solutions instead of the number of students because we are interested in the frequency of certain mistakes.

In this section, $\vec{E}_{I}$ denotes the electric field of the incoming wave while $\overrightarrow{\tilde{E}}_{I}$ was used when using the complex notation. Likewise, $\vec{E}_{R}$ denotes the electric field of the reflected wave and $\vec{E}_{T}$ denotes the electric field of the transmited wave. Similar notations were used for the magnetic field: $\vec{B}_{I}, \vec{B}_{R}$, and $\vec{B}_{T} . \overrightarrow{\widetilde{E}}_{0, I}, \overrightarrow{\widetilde{E}}_{0, R}$, and $\overrightarrow{\widetilde{E}}_{0, T}$ represent the amplitudes of the electric fields of the incoming, reflected and transmitted waves in complex notation, respectively. Similar notations were used for the magnetic field: $\vec{B}_{0, I}, \vec{B}_{0, R}$, and $\vec{B}_{0, T}$.

\section{A. Activation of the tool}

\section{Direct cue (A1)}

If a question not only gives students an explicit prompt but also lists all the equations of $\mathrm{BCs}$, "activation" is considered bypassed. Most exam questions (ES1-4) in the context of EM waves bypass activation. Not only do these questions tell students to use $\mathrm{BCs}$, they also list the equations of BCs explicitly for students. Therefore, in think-aloud interviews, we investigated activation by observing whether students were able to activate $\mathrm{BCs}$ when the equations were not explicitly provided (direct cuewithout equations).

In interviews (IS1), we gave an EM wave problem to three students and asked them to use BCs but did not provide the equations. The question provides a figure similar to Fig. 1 and asks students to write down a mathematical expression relating the amplitude of the electric fields of the incident, reflected, and transmitted waves. Successful activation of BCs involves writing down $\vec{E}_{1}^{\|}=\vec{E}_{2}^{\|}$[Eq. (1)] or any other equations [Eqs. (2)-(6)]. One of the three interviewed students was able to derive the BCs from Maxwell's equations. Two students did not write down the BCs, but rather, jumped straight to this result (with a sign error on $\tilde{E}_{0 R}$ ): $\tilde{E}_{0 I}=\tilde{E}_{0 R}+\widetilde{E}_{0 T}$ (tilde means a complex quantity). A common justification they provided was: 'You can't just come away with more waves than you had coming in. Some of it will go through, some of it will go back. It has to add up, kind of like conservation." Thus, even when asked explicitly to use BCs, but not provided the actual equations, some students did not utilize appropriate BCs; rather they activated a different kind of idea (i.e., the conservation idea). Students were not explicit about whether this "conservation" was the conservation of energy, or the conservation of the amplitude of the electric field, or the conservation of the total reflection and transmitted coefficient (derived from conservation of energy).

\section{Boundary presented (A2)}

Exam set 5 (ES5) did not bypass activation. This question involved two media separated by a boundary (A2) and provided a picture representation like Fig. 1. Students were then asked to determine how the angular frequencies of the incident, reflected, and transmitted waves are related, and to explain or show their reasoning. In a correct solution, setting up the equations of BCs results in an expression in which all the arguments in the exponentials have to be equal in order for the equality to hold at all points along the boundary at all times. Therefore, the angular frequency of the incident wave $\left(\omega_{I}\right)$, reflected wave $\left(\omega_{R}\right)$, and transmitted wave $\left(\omega_{T}\right)$ must all be equal. Most solutions (58 out of 60) correctly indicated that $\omega_{I}=\omega_{R}=\omega_{T}$; however, only half of the solutions (31 out of 60) showed evidence that students had activated BCs. The following scenarios were counted as evidence of activation-mentioning the word "boundary conditions" (4 out of 31) or writing down the equations for BCs (27 out of 31$)$. In the remaining solutions, more than a third (38\%, 23 out of 60) correctly indicated that the angular frequencies are equal but did not explain why. An additional four of the solutions treated the result $\left(\omega_{I}=\omega_{R}=\omega_{T}\right)$ as an a priori fact and stated that $\omega$ is determined by the light source and will not change. Thus, many students appeared to bypass activation of $\mathrm{BCs}$ and instead arrived at their answer using a simple one-step process. We will discuss this further in Sec. VIII. 
In summary, most of our exam questions bypassed activation by asking students to use BCs and listing the equations explicitly. When prompted to use $\mathrm{BCs}$ in the think-aloud interviews (but not provided with the equations), some students did not utilize BCs on the $\vec{E}$ and $\vec{B}$ fields but, instead, used an idea similar to that of "energy conservation." In another set of exam questions which neither prompted students to use BCs nor provided the equations, most students correctly stated the relationship between the angular frequencies of the fields $\left(\omega_{I}=\omega_{R}=\omega_{T}\right)$, but many did not justify this relationship using BCs.

\section{B. Construction of the model \\ 1. Direction of $\vec{k}, \vec{E}$, and/or $\vec{B}$ (C1)}

When constructing a complex expression for the $\vec{E}$ and $\vec{B}$ fields [e.g., Eq. (7)], it is important to know the correct direction for the wave vector $\vec{k}, \vec{E}$, and/or $\vec{B}$. The specific format of the complex expression for the $\vec{E} / \vec{B}$ field depends on the information given in the problem. A general example of what such expressions look like if the wave is polarized in the $x$ direction is given by

$$
\overrightarrow{\tilde{E}}_{I}=\tilde{E}_{0, I} \exp [i(\vec{k} \cdot \vec{r}-\omega t)] \hat{x} .
$$

Here, $\vec{k}$ is the wave vector, $\vec{r}$ is the position vector, $\omega$ is the angular frequency, $t$ is time, and $\hat{x}$ is a unit vector pointing in the $x$ direction.

For this element $(\mathrm{C} 1)$, we focus on exam questions where students were asked to draw the directions of $\vec{k}, \vec{E}$, and/or $\vec{B}$ explicitly. The data sets we use here are exam sets $3 \& 5(N=48$ for ES3, $N=60$ for ES5). ES3 asked for the direction of $\vec{E}_{R}$ when $n_{1}<n_{2}$. ES5 asked students to draw $\vec{E}_{I}$ and $\vec{E}_{T}$ where no specific information about $n_{1}$ and $n_{2}$ was given. Both questions were for normal incidence.

In ES5 $(N=60)$, students were asked to consider a planar wave normally incident from left on the boundary between two linear dielectric media where the wave was polarized in the plane of incidence. The question asks students to draw the directions of the wave vector $\vec{k}$, the (real) electric field $\vec{E}$, and the (real) magnetic field $\vec{B}$ of the incident and the transmitted waves. In students' work, we observed that over a quarter $(28 \%, 17$ out of 60$)$ of the solutions contain errors, most of which are about $\vec{k}$ and $\vec{E}$. The direction of $\vec{B}$ was considered incorrect only if it was inconsistent with the student's $\vec{k}$ and $\vec{E}$ vectors $(N=3)$. Examples of common mistakes included switching the direction of $\vec{E}_{I}$ and $\vec{k}_{I}$ (i.e., $\vec{E}_{I}$ in the direction of propagation and $\vec{k}_{I}$ in the direction of polarization, $N=3$ ), and $\vec{E}_{I}$ pointing in a direction inconsistent with the stated polarization plane $(N=3)$. Four solutions correctly included $\vec{k}_{I}$ pointing perpendicular to the boundary, while having $\vec{k}_{T}$ pointing in an oblique direction. These sets of solutions are different solutions and do not overlap.

In the case of the reflected wave, the direction of $\vec{E}_{R}$ depends on the index of refraction of the two media. For normal incidence when $n_{1}>n_{2}$ (Fig. 1), the direction of $\vec{E}_{R}$ is the same as $\vec{E}_{I}$. When $n_{1}<n_{2}, \vec{E}_{R}$ is out of phase with $\vec{E}_{I}$, meaning $\vec{E}_{R}$ points in the opposite direction. This dependence of the direction of the reflected wave on the relative indices of refraction comes from the Fresnel equations ${ }^{1}$ which are derived from BCs. Thus, it is not possible to know a priori the direction of $\vec{E}_{R}$. In terms of drawing $\vec{E}_{R}$, any preassumed direction is reasonable as long as it is kept consistent within the calculation. On exam set 3 (ES3, $N=48$ ), students were asked explicitly to draw arrows representing the direction of the fields when $n_{1}>n_{2}$. Observations from students' responses show that a third of the solutions $(31 \%, 15$ out of 48$)$ drew the direction of $\vec{E}_{R}$ in the opposite direction of $\vec{E}_{I}$. In most such cases it was not possible to interpret from the written solutions whether students drew $\vec{E}_{R}$ in the opposite direction simply because they were just assuming a direction, intending to let their calculation take care of the sign later. In no cases did we observe direct evidence from the written work that students were making connections between the direction of $\vec{E}_{R}$ and the relationship of the index of refraction between the two media. These data leave open the possibility that some students believe $\vec{E}_{R}$ must point in the opposite direction of $\vec{E}_{I}$ irrespective of the values of refractive index.

\section{Simplifying $\vec{k} \cdot \vec{r}(C 1)$}

For this element, our data sets are exam sets 1 to 4 (ES1-4). ES1 had two questions: one for normal incidence $(N=36)$, and one for oblique incidence $(N=36)$. ES 2, 3, and 4 were all for normal incidence $(N=44,48$, and 30$)$.

Oblique incidence.- - In the case of oblique incidence, the dot product becomes $k_{x} x+k_{y} y+k_{z} z(\mathrm{ES} 1, N=36)$. Half of the solutions (18 out of 36) never wrote down the dot product $\vec{k} \cdot \vec{r}$ at any stage of their solution, but instead jumped to a simple product of the magnitudes of $k$ and $r$, or $k$ and $x, y$, or $z$. Out of those who explicitly calculated the dot product, only 4 worked it out correctly. In the remaining solutions where students at least wrote down $\vec{k} \cdot \vec{r}$, various mistakes were observed including replacing $\vec{k} \cdot \vec{r}$ with $k z$, $k r$, or $k r \sin \theta$.

\footnotetext{
${ }^{1}$ Fresnel equations relate the complex amplitudes of the incident, reflected, and transmitted waves.
} 
Normal incidence.-In the case of normal incidence (ES1-4, $N=128$ ), where $\vec{k}$ is propagating in the $z$ direction (Fig. 1), this dot product simplifies considerably: $\vec{k} \cdot \vec{r}$ becomes $-k_{R} z$ and $k_{T} z$ for the reflected and transmitted waves respectively. However, almost a fifth $(18 \%, 23$ out of 128 ) of the solutions did not include the negative sign for $k_{R}$, and $13 \%$ (16 out of 128, 9 solutions overlap with the previous 23 ) of the solutions did not differentiate between $k_{R}$ and $k_{T}$.

\section{Selecting appropriate equations (C2)}

For this element our data sets are exam sets 1 to 4 (ES1-4). The question on ES1 has two subparts, one for normal incidence $(N=36)$, one for oblique incidence $(N=36)$. ES 2, 3, and 4 are all for normal incidence $(N=44,48$, and 30$)$.

Normal incidence.-Students need to recognize that $\vec{E}$ and $\vec{B}$ are both parallel to the boundary and select the corresponding BCs. Roughly a tenth $(11 \%, 14$ out of 128$)$ of the solutions (ES1-4) chose the wrong BCs equation (using the $\mathrm{BCs}$ for a perpendicular component). One question on the CURrENT (Fig. 2) asks students which of the four equations (i-iv) refers to the $y$ component of the electric field at the interface. The correct answer is (i), although choosing both (i) and (ii) are considered correct as well since both equations refer to the perpendicular component of the electric or magnetic field. On the CURrENT question, $34 \%$ (169 out of 500) got this question wrong, showing that a significant percentage of students have trouble identifying the corresponding components of $\vec{E}$ that are parallel or perpendicular to the boundary.

Oblique incidence.-Students need to determine the correct components of $\overrightarrow{\vec{E}}$ to be used with the corresponding boundary conditions (element C2). A third (33\%, 12 out of 36) of the solutions (ES1) had the wrong components (using sin instead of cos) of the field. Students' written solutions for this data set rarely provided clear evidence to distinguish whether they simply made a basic trigonometry error or if they were, in fact, confused about which component of $\vec{E}$ is parallel vs perpendicular (or indeed whether these terms refer to orientation with respect to the surface or the normal vector to the surface). Therefore, we gave the same question to students in think-aloud interviews (IS1). None of the students from the interviews (IS1, $N=3$ ) with the same question made simple trigonometry errors, suggesting that the former is unlikely to be the dominant issue. Note that these three students all obtained high letter grades (two A's and one A-) in the course, and thus, they may not be representative of all students.

\section{Setting up at the boundary (C3)}

For this element (C3), our data sets are exam sets 1-4 (ES1-4) on normal incidence. Since there exist both incoming and reflected waves in medium 1 (Fig. 1), $\vec{E}$ in medium 1 can be written as $\vec{E}_{I}+\vec{E}_{R}$ (element C3). This is a superposition of two vector quantities and thus the directions need to be considered. As stated earlier in $\mathrm{C} 1$, we do not know the direction of $\vec{E}_{R}$ a priori; it could be in the same direction as $\vec{E}_{I}$ or opposite, depending on the relative indices of refraction. Thus, students can assume any direction for $\vec{E}_{R}$ and their solution would be considered correct as long as they are consistent throughout. Almost $20 \%$ (25 out of 128) of the exam solutions had a superposition expression inconsistent with their choice for the direction of $\vec{E}_{R}$.

Other difficulties were also observed at this step (C3). In order to set up equations at the boundary, students need to replace $\vec{E}_{I}$ and $\vec{B}_{I}$, as well as $\vec{E}_{R}$ and $\vec{B}_{R}$, with the complex expressions obtained previously (C1). In over a third (36\%, 46 out of 128 ) of the solutions (ES1-4), $\vec{E}$ and/or $\vec{B}$ in medium 1 was replaced with the sum of only the amplitudes of the fields: $\tilde{E}_{0, I}+\tilde{E}_{0, R}$ or $\tilde{B}_{0, I}+\tilde{B}_{0, R}$. The exponential terms were lost in these solutions despite the fact that complex forms of the incoming and reflected $\vec{E}$ and $\vec{B}$ were previously obtained. This strategy results in a correct expression only when the boundary is located at $z=0$ (Fig. 1) for normal incidence, while resulting in a wrong expression when the boundary is not at zero (ES2\&3 had $z=d, N=92)$.

We also observed that nearly half of the solutions (57 out of 128, ES1-4) omitted the step of applying the equation at the boundary. Applying the equation at the boundary means replacing the position variable (e.g.: $z$ ) with the specific location of the boundary (e.g., $z_{0}$, where $z_{0}$ can be zero in some problems) and the position variable is contained inside the exponential terms. This omission was observed to happen almost always together with the previous difficulty (exponential terms were lost). Out of these 57 solutions that omitted applying the equation at the boundary, 46 of those also made the mistake of losing the exponential terms. Since the position variable is contained inside the exponential terms, if the exponential terms were lost in an earlier step, the need to plug in the boundary location would be lost as well.

As mentioned previously, a limitation of these written solutions is that they rarely provide evidence for why students make specific errors. However, the spontaneous comments offered by three students on their exam might shed some light on the reasoning. When ignoring the exponential terms and keeping only the amplitude of the $\vec{E}$ field, students arrived at the result: $\left|E_{0, I}\right|=\left|E_{0, R}\right|+\left|E_{0, T}\right|$. They justified their answer as follows: "This makes sense since the incident wave gets partially reflected and partially transmitted." This justification appears similar to the "conservation" reasoning provided by the students interviewed (see previous section of "Activation of the tool"). Similarly, students who offered this justification on the written solution did not explicitly point out which physical 
quantity was being conserved. One hypothesis is the conservation idea might come from students' existing knowledge of energy conservation, which was strongly emphasized in introductory courses. It is possible that due to cognitive interference $[32,33]$ with the idea of energy conservation, students confused their expressions involving electric field amplitudes with the (correct) relation $I_{I}=$ $I_{R}+I_{T}$ (where $I$ represents intensity). However, it would be wrong to assume students simply do not know the difference between intensity and the amplitude of the electric field. As discussed later in this paper (Sec. VII), when explicitly asked about their relationship, students did not treat energy and wave amplitude as interchangeable.

\section{Execution of mathematics}

\section{Simplify at the boundary (E1)}

The most common issue observed in execution is the inappropriate cancellation of all exponential terms. When the boundary is located at $z=0$ (Fig. 1), exponential terms go to 1 , so they can be dropped. However, students still canceled the exponentials when the boundary was located at $z=d$. Over half of the solutions $(54 \%, 50$ out of 92 , ES2\&3) missed the exponential terms in their final answer (see Fig. 4), which was problematic when the boundary had a nonzero location. For some of the written solutions, there was often not enough evidence to tell if students forgot to carry the exponential terms into the boundary conditions (which is a construction error as described in the previous section), or if they simply canceled the exponentials in their head without writing it out on paper (an execution error). Figure 4 shows an example of one such solution. However, out of the 50 solutions that missed the exponential terms, almost half ( 23 out of 50) did show evidence that they were indeed canceling the exponentials (see Fig. 3), suggesting that, for at least some students, this is an execution error rather than a construction error.

The cancellation was coded as an execution error in ACER; however, an execution error does not necessarily mean a pure math mistake. In other words, execution errors are math mistakes made within a physics context, but they may not indicate that the students are incapable of correctly executing the mathematics more generally. In order to further investigate execution errors, we conducted thinkaloud interviews (IS2) with a similar complex exponential expression written in a pure math context. Symbols that

$$
\begin{aligned}
& \tilde{E}_{0}^{I} e^{i(k d-\omega t)} \hat{y}+\left.\tilde{E}_{0}^{R} e^{i(-k d-\omega t+\delta)}\right|_{y}=\tilde{E}_{0}^{T} e^{(k d-\omega t)} \tilde{y} \\
& \tilde{E}_{0}^{N}+\tilde{E}_{0}^{N} e^{i \delta}=\tilde{E}_{0}^{T}
\end{aligned}
$$

FIG. 3. Example of student solution where all the exponential terms were canceled. (Note that the student wrote $I, R$, and $T$ as superscripts instead of subscripts. $\tilde{E}_{0}^{I}$ means the same thing as $\tilde{E}_{0, I}$.)

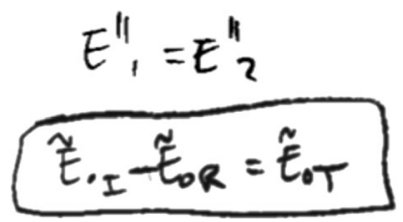

FIG. 4. Example student solution where the nature of the mistake is difficult to determine. There was not enough evidence to tell if students forgot to carry the exponential terms into the boundary conditions (which is a construction error as described in the previous section), or if they simply canceled the exponentials in their head without writing it out on paper (an execution error).

represent physical quantities were replaced with arbitrary math variables (wave vector $k$, speed of light $c$, time $t$ were replaced with $d, a, b)$ :

The following mathematical expression is true when $x=\alpha$ $A e^{i\left(d_{1} x-a b\right)}+B e^{i\left(-d_{1} x-a b\right)}=C e^{i\left(d_{2} x-a b\right)}$

For $x=\alpha$ can you further simplify it?

Even though students made the particular cancellation mistake (execution error in ACER) on the written exam solutions, no students (IS2) made the same cancellation mistake during the think-aloud interviews where the question was given in a pure math context. This finding suggests that this mistake is not due solely to more general problems manipulating exponentials. Interestingly, 3 (out of 4) students still made the connection to the wave context spontaneously: "this is similar to the EM waves problems," "I remember you can always match the coefficients in those boundary condition problems." This suggests students' execution of the mathematics can be affected by the physics context, which may have caused them to cancel all the exponentials during the exam setting.

Even though students in this pure math context did not make the same cancellation mistake, there were some other complex exponential calculations they struggled with. Two students made major math errors with complex exponentials: e.g., $e^{A+B}=e^{A}+e^{B}$ and $e^{-A}=e^{-1} e^{A}$. These results suggest that the use of complex exponentials could be fruitfully explored on its own using ACER in a future investigation.

\section{Mathematical calculation (E2)}

Reflection and transmission coefficients can be calculated using the amplitudes of the $E$ field (E2). Generally students perform well in this ACER element. In exam set 5 (ES5, $N=60$ ), Fresnel equations ${ }^{2}$ were given, and students were asked to calculate the transmission coefficient and represent it in terms of $\alpha$ and $\beta\left(\alpha=\cos \theta_{T} / \cos \theta_{I}\right.$, $\left.\beta=\mu_{1} v_{1} / \mu_{2} v_{2}\right)$. One difficulty students had in this derivation was expressing $\beta$ in terms of $\epsilon_{2} v_{2} / \epsilon_{1} v_{1}$ using the relationship between $\mu, \epsilon$, and $v(22 \%, 13$ out of 60

\footnotetext{
${ }^{2}$ See footnote 1 .
} 
solutions). When writing down the light intensity that is incident on the boundary: almost a fifth (17\%, 10 out of 60) of the solutions omitted $\cos \theta_{I}$ and $\cos \theta_{T}$ from the intensity of the incident and transmitted waves; and a few students (4 out of 60) equate intensity to the modular square of the electric field: $I=|E|^{2}$.

\section{Reflection on the results}

\section{Checking units (R1)}

Evaluating a solution by checking units and limiting behaviors is an important skill that physicists value. Our regular exam questions on BCs did not access this type of "reflection" explicitly, and we saw almost no spontaneous reflection in the written work. Therefore, we constructed an interview question to further investigate how students reflect. We presented students with incorrect but plausible expressions of the reflection and transmission coefficients $R$ and $T$ in a novel situation. Students were asked to examine if these expressions make sense without going through the calculation (see the interview prompt below). Three out of the 4 students interviewed were able to spontaneously point out that the expression of $T$ provided was not unitless (R1).

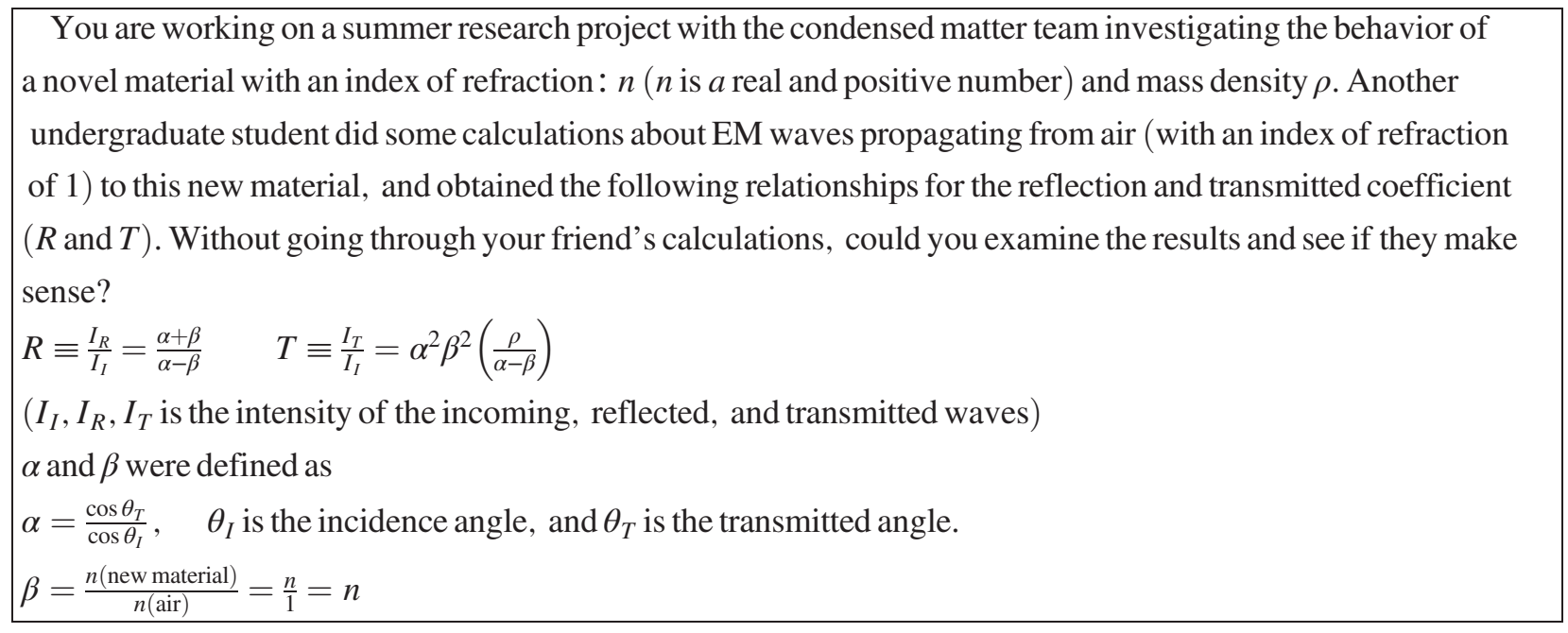

\section{Checking limiting behaviors (R2)}

Compared to unit analysis, students had more difficulty with checking limits. For example, students had trouble identifying the independent variable (incident angle $\theta_{I}$ ). The interviewer had to provide a hint to direct them (3 out of 4) to think about the limits of $R$ and $T$ when $\theta_{I}$ varies from $0^{\circ}$ to $90^{\circ}$. The expressions of $R$ and $T$ given became negative or infinity when taking extreme limits of $\theta_{I}$. Only one student noticed the unphysical limits. Physically, we expect the index of refraction to be larger than the vacuum value of 1 (negative indices [34] were not discussed in this course). One student obtained $T<0$ and did not note that this is unphysical. Two students talked about taking the lower limit of the index of refraction to 0 .
The interview question asks students to evaluate whether a given expression makes sense without providing any structured guidance or further scaffolding. This type of open-ended question requires many skills such as knowing the physical limits of relevant quantities, finding out the limits of one variable as a function of another variable, etc. Our interview suggested that students are struggling with aspects of recognizing the physical limits. The exam questions did not directly target reflection like the interview, but in one case, students were asked a more structured question that revealed similar student difficulties. The exam question gives students Fresnel equations for two different cases and asks students which one can have zero reflection. 
In class and then in homework, we derived the following Fresnel equations, assuming linear media with no

magnetic effects: $E$ field polarized in the plane of incidence

$$
\tilde{E}_{0, R}=\frac{\alpha-\beta}{\alpha+\beta} \tilde{E}_{0, I} \quad \tilde{E}_{0, T}=\frac{2}{\alpha+\beta} \tilde{E}_{0, I}
$$

$E$ field polarized perpendicular to the plane of incidence

$$
\tilde{E}_{0, R}=\frac{1-\alpha \beta}{1+\alpha \beta} \tilde{E}_{0, I} \quad \tilde{E}_{0, T}=\frac{2}{1+\alpha \beta} \tilde{E}_{0, I}
$$

where

$$
\alpha=\frac{\cos \theta_{t}}{\cos \theta_{I}} \quad \text { and } \quad \beta=\frac{n_{2}}{n_{1}}
$$

a) Describe a non-trivial physical situation in which the reflected light would have zero intensity.

b) Describe a non-trivial physical situation in which the electric field vector of the reflected light is 180 degrees out of phase with the $E$ field of the incoming light.

c) Describe a non-trivial physical situation in which the transmitted light would have zero intensity. (Hint: it is possible, you might have to "think outside of the box")

Almost a quarter $(20 \%, 6$ out of 30) of the solutions wrote down unphysical or incorrect limits such as: the index of refraction becomes negative or zero; the velocity of the wave in the media is much larger than the speed of light, $\alpha * \beta=1$ (this cannot happen when $n_{2} \neq n_{1}$ since when $\beta>1, \alpha>1$; when $\beta<1, \alpha<1$ ), etc. In summary, students from the interviews and exams struggled with identifying independent variables, taking the limits of $R$ and $T$, and recognizing unphysical limits.

\section{COMPARISION WITH QUANTUM TUNNELING}

The previous section presented our findings with respect to common student difficulties with BCs in the context of EM waves at an interface. It is also useful to compare student difficulties across subjects to see where there are similarities and/or differences. As mentioned previously, McKagan [11] et al. studied student difficulties with quantum tunneling. A common issue was that students think the energy of the particle is lost after tunneling. One type of reasoning students gave for the energy loss was "since only part of the wave function is transmitted, only part of the energy is transmitted, with the rest being reflected." Therefore, students think that after tunneling, the energy of the particle is lost. The idea that something is "split" between the reflected and transmitted waves also appeared in our study in the context of classical EM waves. In fact, this idea was a significant aspect of student difficulties when applying BCs (discussed previously in Secs. VI A and VI B).

It is possible that students might experience interference of their knowledge and understanding between different contexts. The word "interference" here refers to a cognitive interference (reported previously in cognitive literature $[32,33])$, which occurs in learning when there is an interaction between new material and transfer effects of past learned behavior, memories, or thoughts that have a negative influence in comprehending new material $[32,33]$. Interference in learning quantum mechanics from classical EM waves is understandable because students usually learn the classical waves first and the probability wave idea in quantum is unfamiliar to students (as observed by McKagan et al.). However, interference from quantum mechanics while learning classical EM waves is also possible, especially if students are taking these two courses at the same time. In our study, we observed one example of such interference. When a student was trying to decide if there should be a plus or minus sign between $\vec{E}_{I}$ and $\vec{E}_{R}$, they wrote down $1=\operatorname{Prob}(R)+\operatorname{Prob}(T)$, stating that the total probability of reflection and transmission should add up to be 1 . Then the student continued to write: $1-\operatorname{Prob}(R)=\operatorname{Prob}(T)$, and then confirmed that there should be a minus sign before the $E$ field of the reflected wave. The student concluded that the answer should be: $\vec{E}_{I}-\vec{E}_{R}=\vec{E}_{T}$. When asked by the interviewer what they meant by "probability", the student said: "I'm also taking quantum mechanics and I remember those from my quantum class."

Another reason students gave for energy loss in quantum tunneling was that they were thinking about the relationship between energy and amplitude (the two are directly related in classical situations) [11]. Previous studies have indicated that students' understanding of the energyamplitude relationship in the classical sense hinders their understanding of the probability waves in the new context (i.e., quantum mechanics). Interestingly, even in the context of classical EM waves, we found that students' understanding of the energy-amplitude relationship could also affect their performance with BCs problems. From both the exam solutions and student interviews, we observed that students often wrote down $\left|E_{0, I}\right|=\left|E_{0, R}\right|+\left|E_{0, T}\right|$, and justified this relationship using an idea similar to a 
conservation idea: part of the wave is reflected and part of the wave is transmitted. One hypothesis is students confused their expressions involving electric field amplitudes with the relation involving intensities: $I_{I}=I_{R}+I_{T}$; this is likely a cognitive interference [32,33] with the idea of energy conservation. Here, in a classical electricity and magnetism context, we observed that students' understanding of the energy-amplitude relationship could be hindering them from applying BCs correctly.

However, when being explicitly asked about their relationship, students did not treat energy and wave amplitude as interchangeable (only one student wrote down $I=|E|$ on ES5). It is possible that when being prompted explicitly to calculate the transmission coefficient, students are reminded to check for the definition of the physical quantity "intensity" and therefore did not equate $I$ to $|E|$. While not being a focus of this paper, research regarding students' understanding of energy-amplitude relationship could be an interesting future project.

\section{CONCLUSIONS AND DISCUSSIONS}

BCs appear in different contexts throughout in the advanced undergraduate curriculum: classical mechanics, quantum mechanics, and throughout electricity and magnetism. There has been relatively little research on student difficulties with BCs. This paper contributes to this body of research on analysis of student problem solving using BCs by focusing on the context of EM waves in junior-level electrodynamics. The analysis was guided by the ACER framework that provides an organizing structure that helped us identify points in students' work where key difficulties appear. It also informed the development of interview protocols that further helped us investigate student reasoning.

Here we summarize our findings with student difficulties organized by the ACER framework.

(i) Activation: Our regular exam questions tended to bypass activation by listing BCs explicitly for students and asking them to use BCs. In the absence of such direct activation (think-aloud interviews), some students had trouble activating BCs but instead activated an idea similar to that of "energy conservation." When an exam question gave students a physical situation involving two media and asked for the relationship between the physical quantity (e.g., angular frequency $\omega$ ) across the boundary, students mostly did not have any trouble knowing the correct relationship $\left(\omega_{I}=\omega_{R}=\omega_{T}\right)$; however, many did not justify this relationship using BCs.

(ii) Construction: Writing complex expressions for $\vec{E}$ and/or $\vec{B}$ field of the incoming, reflected, and transmitted waves is an important construction element. Common student difficulties with this element include the following: Finding the correct direction for $\vec{E}_{R}$ and simplifying the dot product $\vec{k} \cdot \vec{r}$. Other common difficulties with construction include choosing the correct components for the parallel and perpendicular fields; and setting up equations only at the boundary.

(iii) Execution: Students were observed to cancel out exponential terms inappropriately even when the boundary was not located at zero. This execution issue was not simply a math mistake, since students did not make the same mistake in a pure math context. However, interviews showed that students were still struggling with other aspects of conducting and simplifying complex exponential math.

(iv) Reflection: Our exam questions rarely asked students to reflect, and we observe few spontaneous reflections. We constructed interview questions to investigate reflection. Students' difficulties included checking the physical limits of the reflected and transmitted coefficient when asked to evaluate the reasonableness of their results.

These findings have several potential implications for teaching and assessing the use of BCs in electrodynamics and even across subjects. In our physics class, students often only practice on simple situations (e.g., boundary $z=0$ ) and this may cause them to overgeneralize the results to other situations. It is likely worthwhile to present students a variety of physical situations where there can be both simple and messier results. Students could also benefit from practice recognizing unphysical limits and taking explicit limits using corresponding independent variables to develop their reflection skills.

This study has several important limitations. We only focus here on the use of BCs without exploring the use of complex exponentials by itself, which is also an important aspect of solving most of these BCs questions. Another limitation was inherited from the analytical framework (ACER) itself. ACER does not explicitly address the nuance associated with representations. For example, the framework does not explore the translation between verbal, mathematical, graphical, and pictorial representations, which is almost always required to solve physics problems [13]. Furthermore, the statistics provided from the written solutions about student difficulties at different points of the ACER framework provide information on the frequency of particular errors, but often do not provide detail on the reasoning mechanism behind these errors. To address this, the findings from the exam solution analysis informed us on the development of interview protocols that help us further investigate the mechanism of student reasoning. In addition, our analysis showed that sometimes students took a different approach by activating a different type of tool instead of BCs in solving the problem, effectively short circuiting their problem solving process, e.g., making their solution a simple one-step solution instead of a multiplestep process. For example, when students were asked to 
find the relationship among the angular frequencies of the incident, reflected, and transmitted waves, many students did not activate BCs, but instead treated the (correct) result as an a priori fact. This type of shortcut bypasses the rest of the operationalized ACER framework and limits what we can learn from these solutions. This also happened when students activated the idea of conservation and arrived at a result that does not require any additional algorithmic process. Therefore, the construction and execution pieces of the framework of ACER become nonapplicable to that student.

It has been reported elsewhere that students' epistemological framing of the problem can impact how they answer questions [35]. For example, students who enter an algorithmic math frame [35] would spend a considerable amount of time setting up a series of algebra-based steps such as evaluating integrals, taking derivatives, etc. Students in a conceptual physics frame [35] would try to think in terms of the features of the physical system and might coordinate between different representations such as graphical, geometric, or gestural to visualize the physical system. It is possible that what kind of tools students activate could affect their framing of the question. The problem text could also affect or direct students in a certain way. We did not study students' epistemological framings in this paper; however, an interesting future research direction could look at the relationship between students' epistemological framings and the mathematical or physical tools they choose to activate.

Our ongoing projects involve analyses of student difficulties with BCs using the theoretical framework of symbolic forms $[22,23]$, which will shed light on students' reasoning mechanisms. We also plan to expand the investigation of student difficulties across different contexts: in both electricity and magnetism and quantum mechanics. Further work could also include taking the richness of using different representations into consideration. We also plan to further investigate student difficulties on the use of complex exponentials as a mathematical tool, in order to compare with student difficulties in the use of this physical concept (i.e., BCs).

\section{ACKNOWLEDGMENTS}

We gratefully acknowledge the contributions of Andreas Becker, Dimitri Dounas-Frazer, and the PER@C group. This work was supported by NSF-CCLI Grant No. 1023208.
[1] D. E. Meltzer and R. K. Thornton, Resource letter alip1: Active-learning instruction in physics, Am. J. Phys. 80, 478 (2012).

[2] M. D. Caballero, B. R. Wilcox, L. Doughty, and S. J. Pollock, Unpacking students use of mathematics in upper-division physics: Where do we go from here?, Eur. J. Phys. 36, 065004 (2015).

[3] R. E. Pepper, S. V. Chasteen, S. J. Pollock, and K. K. Perkins, Observations on student difficulties with mathematics in upper-division electricity and magnetism, Phys. Rev. ST Phys. Educ. Res. 8, 010111 (2012).

[4] C. A. Manogue, P. J. Siemens, J. Tate, K. Browne, M. L. Niess, and A. J. Wolfer, Paradigms in physics: A new upper-division curriculum, Am. J. Phys. 69, 978 (2001).

[5] M. Boas, Mathematical Methods in the Physical Sciences, 2nd ed. (Wiley, New York, 1983).

[6] J. Taylor, Classical Mechanics (University Science Books, Sausalito, CA, 2005).

[7] D. Griffiths, Introduction to Electrodynamics, 3rd ed. (Prentice-Hall, Inc., Upper-Saddle River, NJ, 1999).

[8] B. R. Wilcox and S. J. Pollock, Upper-division student difficulties with separation of variables, Phys. Rev. ST Phys. Educ. Res. 11, 020131 (2015).

[9] C. Singh and E. Marshman, Review of student difficulties in upper-level quantum mechanics, Phys. Rev. ST Phys. Educ. Res. 11, 020117 (2015).
[10] C. Singh, Student understanding of quantum mechanics at the beginning of graduate instruction, Am. J. Phys. 76, 277 (2008).

[11] S. B. McKagan, K. K. Perkins, and C. E. Wieman, Deeper look at student learning of quantum mechanics: The case of tunneling, Phys. Rev. ST Phys. Educ. Res. 4, 020103 (2008).

[12] J. P. Zwolak and C. A. Manogue, Assessing student reasoning in upper-division electricity and magnetism at oregon state university, Phys. Rev. ST Phys. Educ. Res. 11, 020125 (2015).

[13] B. R. Wilcox, M. D. Caballero, D. A. Rehn, and S. J. Pollock, Analytic framework for students' use of mathematics in upper-division physics, Phys. Rev. ST Phys. Educ. Res. 9, 020119 (2013).

[14] B. R. Wilcox and S. J. Pollock, Upper-division student difficulties with the dirac delta function, Phys. Rev. ST Phys. Educ. Res. 11, 010108 (2015).

[15] B. Wilcox and S. J. Pollock, Student difficulties with delta functions, Proceedings of the Physics Education Research Conference 2014, Minneapolis, MN (AIP, New York, 2014), pp. 271-274.

[16] D. Hammer, Student resources for learning introductory physics, Am. J. Phys. 68, S52 (2000).

[17] J. Tuminaro, A cognitive framework for analyzing and describing introductory students' use and understanding of mathematics in physics, Ph.D. thesis, University of Maryland, 2004. 
[18] J. Tuminaro and E. F. Redish, Elements of a cognitive model of physics problem solving: Epistemic games, Phys. Rev. ST Phys. Educ. Res. 3, 020101 (2007).

[19] B. Tuminaro, An epistemic framing analysis of upperlevel physics students' use of mathematics, Ph.D. thesis, University of Maryland, 2008.

[20] A. diSessa, Towards an epistemology of physics, Cognit. Instr. 10, 105 (1993).

[21] A. diSessa and B. Sherin, What changes in conceptual change?, Int. J. Sci. Educ. 20, 1155 (1998).

[22] B. Sherin, The symbolic basis of physical intuition: A study of two symbol systems in physics instruction, Ph.D. thesis, University of California, Berkeley, 2008.

[23] A. diSessa and B. Sherin, How students understand physics equations, Cognit. Instr. 19, 479 (2001).

[24] A. Collins and W. Ferguson, Epistemic forms and epistemic games: Structures and strategies to guide inquiry, Educ. Psychol. 28, 25 (1993).

[25] B. R. Wilcox, New tools for investigating student learning in upper-division electrostatics (2015), https://scholar .colorado.edu/phys_gradetds/135.

[26] S. V. Chasteen, S. J. Pollock, R. E. Pepper, and K. K. Perkins, Transforming the junior level: Outcomes from instruction and research in E\&M, Phys. Rev. ST Phys. Educ. Res. 8, 020107 (2012).

[27] E. Mazur, Peer Instruction: Users Manual, Series in Educational Innovation (Prentice Hall, Upper Saddle River, NJ, 1997).
[28] C. Baily, M. Dubson, and S J. Pollock, Research-based course materials and assessments for upper-division electrodynamics (e\&m ii), Proceedings of the Physics Education Research Conference 2012, Philadelphia, PA (AIP, New York, 2012), pp. 54-57.

[29] Q. X. Ryan, C. Astolfi, C. Baily, and S. J. Pollock, Validation of a conceptual assessment tool in e\&m ii, Proceedings of the Physics Education Research Conference 2014, Minneapolis, MN (AIP, New York, 2014), pp. 231-234.

[30] http://www.colorado.edu/physics/EducationIssues/ Electrodynamics/.

[31] S. Mayer, The early evolution of jean Piaget's clinical method, Hist. Psychol. 8, 362 (2005).

[32] J. T. Wixted, The psychology and neuroscience of forgetting, Annu. Rev. Psychol. 55, 235 (2004).

[33] T. D. Tomlinson, D. E. Huber, C. A. Rieth, and E. J. Davelaar, An interference account of cue-independent forgetting in the no-think paradigm, Proc. Natl. Acad. Sci. U.S.A. 106, 15588 (2009).

[34] S. Zhang, Y.-S. Park, J. Li, X. Lu, W. Zhang, and X. Zhang, Negative Refractive Index in Chiral Metamaterials, Phys. Rev. Lett. 102, 023901 (2009).

[35] B. Modir, J. D. Thompson, and E. C. Sayre, Students' epistemological framing in quantum mechanics problem solving, Phys. Rev. Phys. Educ. Res. 13, 020108 (2017). 